4. Schweiger T, Nenekidis I, Stadler JE, Schwarz S, Benazzo A, Jaksch P, et al. Single running suture technique is associated with a low rate of bronchial complications after lung transplantation. J Thorac Cardiovasc Surg. 2020;160: 1099-108.e3.
5. Crespo MM, McCarthy DP, Hopkins PM, Clark SC, Budev M, Bermudez CA, et al. ISHLT consensus statement on adult and pediatric airway complications after lung transplantation: definitions, grading system, and therapeutics. J Heart Lung Transplant. 2018;37:548-63.
See Article page 1099.

\section{Commentary: Running bronchial anastomotic suture in lung transplantation: Should we run before we walk?}

\author{
Jules Lin, MD
}

Schweitzer and colleagues ${ }^{1}$ report a large series of 1555 patients undergoing lung transplant using a single running suture bronchial anastomotic technique. Although many surgeons are hesitant to use a running suture due to the risk of purse-stringing and narrowing the anastomosis, the authors should be congratulated on achieving excellent results with a low rate of anastomotic complications. These complications have decreased over time, and the majority were treated endoscopically.

Including patients over almost 2 decades allowed the authors to create a larger study cohort, but the long time period introduces potential confounding issues. Changes in lung transplant selection and allocation (eg, distance, primary disease group, disease severity, and functional status), anastomotic and operative technique (eg, donor bronchial length, extracorporeal membrane oxygenation [ECMO], or cardiopulmonary bypass), donor procurement (eg, donation after cardiac death, retrograde perfusion, preservation solutions, or ex vivo lung perfusion [EVLP]), postoperative care, and immunosuppression (induction therapy) could all potentially affect the incidence of anastomotic complications.

In the current study, the disease group, use of ECMO intraoperatively and as a bridge-to-transplant, and EVLP

From the Section of Thoracic Surgery, Department of Surgery, University of Michigan Medical Center, Ann Arbor, Mich.

Disclosure: Author has nothing to disclose with regard to commercial support.

Received for publication March 1, 2020; accepted for publication March 3, 2020; available ahead of print March 19, 2020.

Address for reprints: Jules Lin, MD, Section of Thoracic Surgery, Department of Surgery, University of Michigan Medical Center, 1500 E Medical Center Dr, 2120TC/ 5344, Ann Arbor, MI 48109-5344 (E-mail: juleslin@umich.edu).

J Thorac Cardiovasc Surg 2020;160:1110-1

$0022-5223 / \$ 36.00$

Copyright (c) 2020 by The American Association for Thoracic Surgery

https://doi.org/10.1016/j.jtcvs.2020.03.022

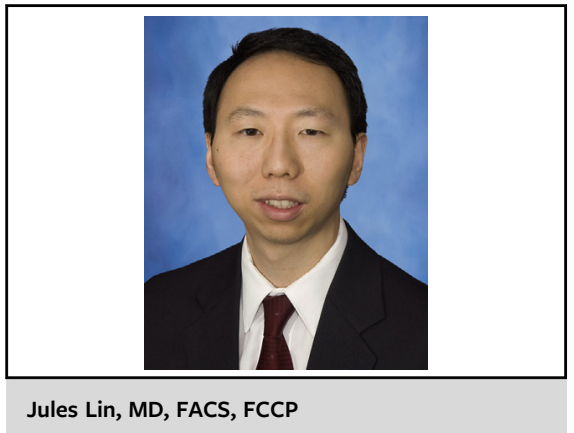

CENTRAL MESSAGE

Although many surgeons hesi-

tate to use a single running su-

ture bronchial anastomotic

technique, this large series re-

ports a low rate of bronchial

complications.

changed significantly over time. However, there was no association between ECMO or EVLP and bronchial complications, and the authors believe that the lower complication rate during the later time periods was due to increasing experience and transplant volumes. Anastomotic complications were higher with antithymoglobulin and with no induction therapy, and the majority of these patients were transplanted in the earlier time periods. Those treated with antithymoglobulin or without induction received higher doses of corticosteroids during the first year, which has been associated with anastomotic complications.

Anastomotic complications were higher with lobar transplants with 7 of the 45 airway complications in the lobar group. The incidence for anastomotic complications was $3.3 \%$ (7 out of 212) after lobar transplant, and all were right-sided. Mitilian and colleagues ${ }^{2}$ also found a higher anastomotic complication rate, with $16 \%$ requiring endoscopic treatment. In addition, airway complications may have a greater influence on the outcomes of lobar lung transplant recipients. ${ }^{3}$ 
Other factors previously associated with airway complications include positive airway cultures pre and posttransplant, primary graft dysfunction, and ischemic time. $^{4,5}$ However, the authors found no association between mechanical ventilation $>48$ hours, mean ischemic time $\geq 345$ minutes, postoperative ECMO, or primary graft dysfunction 2 or 3 at 72 hours and airway complications. A recent review by Anile and colleagues ${ }^{6}$ on bronchial anastomoses for lung transplant included three studies with a partial or complete running anastomotic technique with a rate of airway complications ranging from $2.1 \%$ to $23.8 \%$. Absorbable sutures, a short donor bronchus, and avoiding telescoping the anastomosis were associated with lower anastomotic complication rates.

There have been concerns in the past that a running bronchial anastomotic suture could result in purse-stringing and narrowing of the anastomosis; however, the authors' large experience with a low complication rate using a running technique suggests that in this case, it may be safe to run before we walk.

\section{References}

1. Schweiger T, Nenekidis I, Stadler JE, Schwarz S, Benazzo A, Jaksch P, et al. Single running suture technique is associated with low rate of bronchial complications after lung transplantation. J Thorac Cardiovasc Surg. 2020;160: 1099-108.e3.

2. Mitilian D, Sage E, Puyo P, Bonnette P, Parquin F, Stern M, et al. Techniques and results of lobar lung transplantations. Eur J Cardiothorac Surg. 2014;45:365-9.

3. Sugimoto S, Yamane M, Otani S, Kurosaki T, Okahara S, Hikasa Y, et al. Airway complications have a greater impact on the outcomes of living-donor lobar lung transplantation recipients than cadaveric lung transplantation recipients. Surg Today. 2018;48:848-55

4. Olland A, Reeb J, Puyraveau M, Hirschi S, Seitlinger J, Santelmo N, et al Bronchial complications after lung transplantation are associated with primary lung graft dysfunction and surgical technique. J Heart Lung Transplant. 2017 36:157-65.

5. Yserbyt J, Dooms C, Vos R, Dupont LJ, Van Raemdonck DE, Verleden GM. Anastomotic airway complications after lung transplantation: risk factors, treatment modalities and outcome - a single-centre experience. Eur J Cardiothorac Surg. 2016;49:e1-8.

6. Anile M, Diso D, Rendina EA, Venuta F. Airway anastomosis for lung transplantation. J Thorac Dis. 2016;8(Suppl 2):S197-203. 